\title{
THE ROLE OF PELLET THERMAL STABILITY IN REACTOR DESIGN FOR HETEROGENEOUSLY CATALYSED CHEMICAL REACTIONS
}

\author{
R. J. WIJNGAARDEN ${ }^{\dagger}$ and K. R. WESTERTERP ${ }^{\dagger}$ \\ Chemical Reaction Engineering Laboratories, Department of Chemical Engineering, Twente University, \\ P.O. Box 217, 7500 AE Enschede, The Netherlands
}

(Received 25 June 1990; accepted for publication 3 September 1991)

\begin{abstract}
For exothermic fluid-phase reactions, a reactor which is cooled at the wall can exhibit multiplicity or parametric sensitivity. Moreover, for heterogeneously catalysed exothermic fluid-phase reactions, each of the catalytically active pellets in the reactor can exhibit multiplicity. Both forms of multiplicity can lead to thermal instability and as such have to be taken into account in reactor design. Here the effect of both instabilities is quantified. To this end, simple first-order kinetics are assumed, and intraparticle resistances and reactor and particle dynamics are not considered. $\mathbf{A}$ one-dimensional model, consisting of microscale mass and heat balances, is chosen to describe the reactor. It is assumed that the fluid inlet temperature equals the coolant temperature. The pellet scale model is a combined mass and heat balance for the pellet and it assumes that the Chilton-Colburn analogy holds. For its incorporation in the reactor model it is assumed that for every individual pellet heat removal to neighbouring pellets via the mutual contact spots is negligible as compared to the heat transferred to the surrounding fluid. Consequently every pellet is isolated from its neighbours. In the thermally most critical region, i.e. the hot-spot region, reactor stability is determined by three parameter groups: a dimensionless adiabatic temperature rise, an Arrhenius number or dimensionless activation temperature and the ratio of the number of heat transfer units to the number of reaction units. For pellet multiplicity, a fourth parameter group becomes significant in addition: the ratio of the reaction rate to the pellet mass transfer rate. This number depends on the pellet size. A general recipe is given which enables us to determine whether or not pellet thermal instability can become important in reactor operation. For the situation where it is sigmificant, generalized diagrams are presented indicating which pellet sizes problems must be expected due to pellet multiplicity.
\end{abstract}

\section{INTRODUCTION}

The basic principles of reactor multiplicity or, depending on the model used, of parametric sensitivity and pellet multiplicity can be found in most text books [see Westerterp et al. (1987)]. For heterogeneous exothermic reactions run away occurs either at reactor or pellet level. Below we shall refer to the first type of run away as a reactor run away; the latter type will be referred to as pellet run away.

If a certain fraction of the pellets in the reactor can operate either at a lower or a higher stable operating point and these pellets are thermally isolated from each other, there will be a large number of operating points possible for the reactor. This phenomenon is referred to as infinite multiplicity, first pointed out by Liu and Amundson $(1962,1963)$ and Liu et al. (1963). If we have axial dispersion of heat in the reactor, infinite multiplicity will not occur, because, if one individual pellet in the reactor operates at the higher operating point, its temperature will rise rapidly, and, via axial dispersion-through the fluid or solid pellet phase-and via forced convection, the neighbouring pellets will be heated up, up to the point where they also are forced to switch to the higher stable operating point. These pellets, in turn, heat up their neighbour-

\footnotetext{
† Present address: Koninklijke/Shell-Laboratorium, Amsterdam, P.O. Box 3003, 1003 AA Amsterdam, The Netherlands.

tAuthor to whom correspondence should be addressed.
}

ing pellets and eventually a pellet run-away front will travel through the packed bed until its boundaries are reached and the run away is complete. Consequently there are only two operating regimes: either all pellets are at the lower operating point or they are all at the higher operating point and for the latter case it is sufficient that only one pellet switches to or operates at its higher stable operating point.

The line of reasoning above was first discussed by Eigenberger (1972), who showed quantitatively that infinite multiplicity will not occur if there is even a small amount of axial dispersion via the solid phase. Essentially the same reasoning is used here, albeit that in our opinion axial dispersion need not necessarily occur via the solid phase: it may also occur via the interstitial fluid phase or any combination of both phases will do. Here we neglect the amount of heat transferred between two neighbouring pellets via their contact spot. This may seem in contradiction with the line of reasoning adopted by Eigenberger (1972), but it is immaterial whether heat is transferred to the neighbouring particles via the gas phase or via dispersion in the solid phase.

For exothermic reactions where we have adiabatic temperature rises of hundreds to thousand(s) of Kelvin, we typically operate at lower stable operating points, both for the reactor and for all pellets contained in it. If we have either a reactor or pellet run away, this run away will automatically force the other type of run away to occur because of the high temper- 
atures involved. For example, if a reactor run away occurs, the temperature of the fluid surrounding the pellets becomes so high that automatically all pellets in the reactor are forced to operate at their higher stable operating point. On the other hand if one pellet exhibits run away it will initiate all other pellets in the reactor as discussed above, resulting in extremely high bed temperatures which force the reactor to operate in its higher operating regime.

Therefore, if a run away is established, it will be thereafter impossible to distinguish which phenomenon initiated the temperature rise. Yet the distinction between them is very important. If the run away is caused by pellet run away, in order to prevent it the pellet size must be decreased, thereby enhancing heat removal from the pellet to the fluid. On the other hand, if reactor runaway is the cause, the pellet size must be increased, thereby increasing the effective radial heat conductivity of the packed bed and thus improving heat removal from the reactor to the coolant. Hence, to prevent a possible run away here, both types of run away force us to take an opposite action. Consequently it must be expected that both run-away types also have a different impact in reactor design, as will be quantified here.

The models discussed below are all steady-state models. Hence, forms of instability other than multiplicity and parametric sensitivity, e.g. limit cycles and chaotic behaviour, are not considered. Furthermore intraparticle resistances are neglected and first-order kinetics are assumed. Morbidelli and Varma (1986, 1987) include intraparticle resistances and $n$ th-order kinetics in their stability analysis. The results obtained are similar to the ones derived here, but are more complicated and therefore less accessible. Here we shall present simple equations which can be used for a quick check of the significance of pellet thermal instability.

\section{REACTOR AND PELLET MODELS}

We shall give a brief description of the reactor and pellet models and assume in both cases simple firstorder kinetics.

\section{Reactor model}

The reactor model used is one dimensional. It is assumed that axial dispersion of mass is negligible as compared to axial convection. Axial dispersion of heat is only important insofar as it eliminates infinite multiplicity. Hence, if as much as one pellet exhibits run away we shall assume that this leads to reactor run away. Further, the effect of axial dispersion of heat on the axial temperature profile can be neglected compared to axial convection. Now a microscale mass balance yields

$$
\frac{\partial \Gamma}{\partial \omega}=+(1-\Gamma) \exp \left(\frac{\psi \Theta}{1+\Theta}\right)
$$

A heat balance gives

$$
\frac{\partial \Theta}{\partial \omega}=+\Delta \Theta_{a d}(1-\Gamma) \exp \left(\frac{\psi \Theta}{1+\Theta}\right)-\Xi \Theta .
$$

For $\Xi=0$ we have an adiabatic packed-bed reactor; for $\Xi=\infty$ we have an isothermal packed bed and the temperature equals the coolant temperature everywhere in the packed bed.

The above equations are solved subject to the initial conditions

$$
\omega=0 \Rightarrow \Gamma=0
$$

stating no conversion at the entrance,

$$
\omega=0 \Rightarrow \Theta=0
$$

i.e. the fluid inlet temperature equals the coolant temperature.

Notice that in this model the reactor length is not incorporated. We assume that the thermally most dangerous location-for cooled tubular reactors the hot spot-is located inside the reactor. At this location, chances to get a pellet run away are highest. Furthermore, if a reactor run away is initiated it will also occur here. Since we only investigate the effect of pellet instability on reactor operation, any part of the reactor situated beyond this location is of no significance to us. Consequently if this spot is situated inside the reactor, the reactor length becomes insignificant and we do not need it in our description. Thus we can discard the reactor length as a design variable, which simplifies representation of the results considerably.

\section{Pellet model}

To arrive at the pellet model it is assumed that intraparticle concentration and temperature gradients are negligible. Combining the pellet mass and heat balances and adopting the Chilton-Colburn analogy a final solution is found which can be written as

$$
\theta_{p}-\theta_{f}=\frac{\phi \exp \left(-\frac{\theta_{a}}{\theta_{p}}\right)}{1+\phi \exp \left(-\frac{\theta_{a}}{\theta_{p}}\right)} .
$$

The dimensionless numbers in the above equation were chosen such that pellet multiplicity can be described with a minimum number of parameters. The left-hand side of eq. (5) can be regarded a dimensionless pellet heat withdrawal rate, and the righthand term represents a corresponding heat production rate. Solving for $\theta_{p}$, for fixed values of $\theta_{f}, \theta_{a}$ and $\phi$, we find either one, two or three solutions. In the latter cases we have pellet multiplicity. If such is the case and we increase the pellet size (i.e. $\phi$ ), with $\theta_{r}$ and $\theta_{a}$ remaining constant, for a certain critical pellet size the pellet specific heat transfer area becomes too low and the pellet is forced to switch from its lower operating point to the higher. This we refer to as pellet run away. From eq. (5) the critical value of $\phi$ above which run away will occur, $\phi_{r a}$, can be calculated by solving 
the following set of equations:

$$
\left.\begin{array}{c}
\frac{\phi_{r a} \exp \left(-\frac{\theta_{a}}{\theta_{p}}\right)}{1+\phi_{r a} \exp \left(-\frac{\theta_{a}}{\theta_{p}}\right)}=\theta_{p}-\theta_{f} \\
\frac{\partial}{\partial \theta_{p}}\left[\frac{\phi_{r a} \exp \left(-\frac{\theta_{a}}{\theta_{p}}\right)}{1+\phi_{r a} \exp \left(-\frac{\theta_{a}}{\theta_{p}}\right)}\right]=1
\end{array}\right\} .
$$

This last relation says that the slopes of the heat production rate (HPR) curve and the heat withdrawal rate (HWR) line at the operating point in a HPR/HWR versus pellet temperature diagram are equal.

This yields the following result:

$$
\begin{aligned}
\phi_{r a}= & \frac{\theta_{a}-2 \theta_{f}-\sqrt{\theta_{a}\left(\theta_{a}-4 \theta_{f}-4 \theta_{f}^{2}\right)}}{2+\theta_{a}+2 \theta_{f}+\sqrt{\theta_{a}\left(\theta_{a}-4 \theta_{f}-4 \theta_{f}^{2}\right)}} \\
& \times \exp \left[\frac{2 \theta_{a}\left(1+\theta_{a}\right)}{\theta_{a}+2 \theta_{a} \theta_{f}-\sqrt{\theta_{a}\left(\theta_{a}-4 \theta_{f}-4 \theta_{f}^{2}\right)}}\right] .
\end{aligned}
$$

If $\phi>\phi_{r a}$ we have pellet multiplicity; if $\phi \leqslant \phi_{r a}$ the pellet remains at its lower stable operating point. For

$$
\theta_{a}<4 \theta_{f}\left(1+\theta_{f}\right)
$$

pellet run away cannot occur for any value of $\phi$, so in this case eq. (7) is inapplicable.
COMBINATION OF REACTOR AND PELLET MODELS

We observe that we use two different dimensionless temperatures $\Theta$ and $\theta$. We have done so to obtain a description of both reactor and pellet multiplicity with a minimum number of parameters. Now the parameters $\Theta$ and $\theta$ will be related to each other. By combination of the definitions of the dimensionless numbers, $\theta_{f}$ and $\theta_{a}$ can be calculated for every location in the reactor according to

$$
\theta_{f}=\frac{1+\Theta}{(1-\Gamma) \Delta \Theta_{\alpha d}} L e^{2 / 3}
$$

and

$$
\theta_{a}=\frac{\psi}{(1-\Gamma) \Delta \Theta_{a d}} L e^{2 / 3}
$$

Substitution of the above equations into eq. (7) gives $\phi_{\text {ra }}$ for any given location $\omega$. This is illustrated in Fig. 1.

Pellet multiplicity will not occur in a region in the reactor if for this region

$$
\psi<4(1+\Theta)\left[1+\frac{1+\Theta}{(1-\Gamma) \Delta \Theta_{a d}} L e^{2 / 3}\right]
$$

as can be seen by combining eqs (8), (9) and (10). Since for $\omega \rightarrow \infty$ we have $\Gamma \rightarrow 1$ and $\Theta \rightarrow 0$, condition (11) is always fulfilled for sufficiently high $\omega$ values. Thus, there must always be a location in the reactor from which point on pellet multiplicity cannot occur anymore. This point is indicated by a cross in Fig. 1.

Here we are interested in the minimum value of
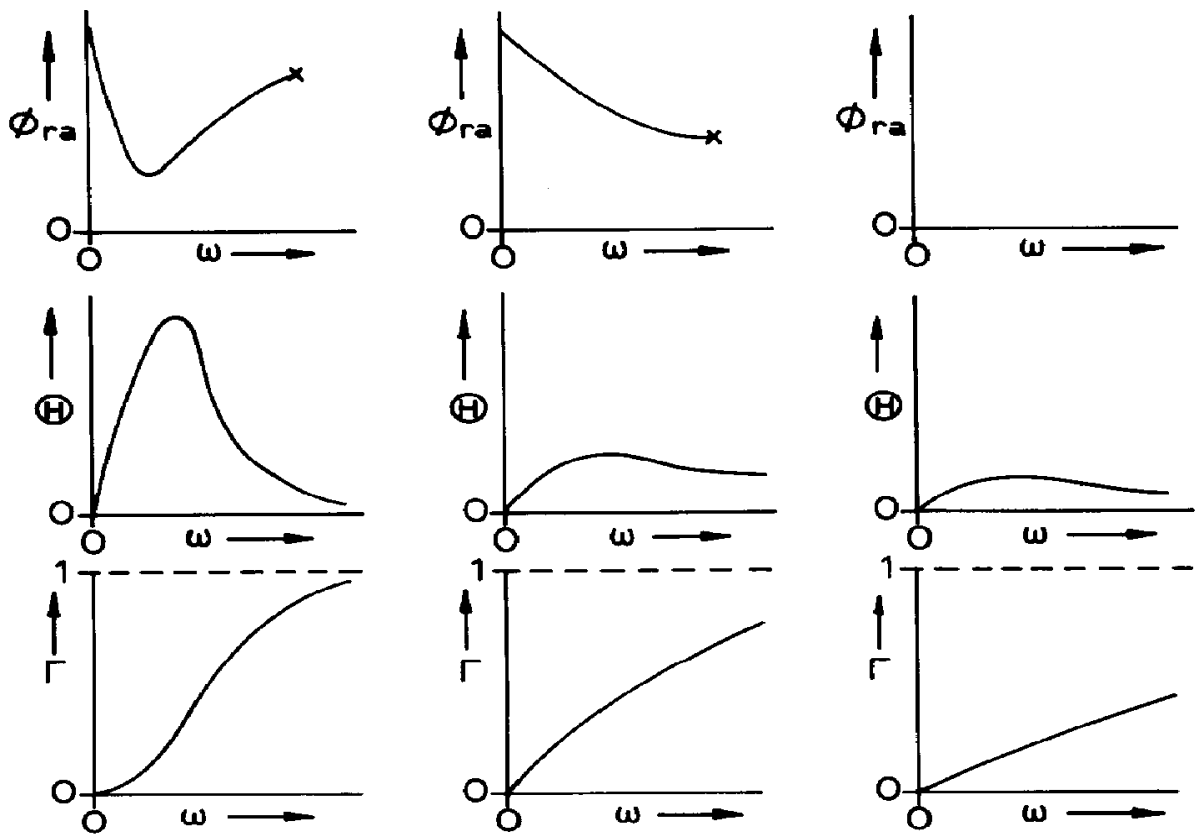

Fig. 1. Sketches of the dimensionless pellet size for which run away occurs; $\phi_{\text {ra, }}$ the dimensionless bed temperature $\Theta$ and the conversion $\Gamma$ versus the dimensionless bed height $\omega$. 
$\phi_{r a}$ in the reactor:

$$
\phi_{\text {ra, min }}=\min _{\infty \in[0, \infty)} \phi_{r a} .
$$

This parameter gives us the pellet size below which no pellet run away can occur at any location in the reactor. Hence, the pellet size used in the reactor design must be smaller than the value calculated from $\phi_{r a, \min }$ :

$$
\left(\frac{V_{p}}{A_{p}}\right)_{\mathrm{design}}<\frac{k_{f}}{k_{\mathrm{o}}} \phi_{\mathrm{ra}, \min } .
$$

For the determination of $\phi_{r a, \text { min }}$ three regimes must be distinguished (see Fig. 1)

(i) A distinct minimum is found for $\phi_{r a}$ before the point where pellet multiplicity vanishes. The location where this minimum occurs will be just before the position where the highest temperature is found in the reactor: the hot-spot region.

(ii) Pellet multiplicity vanishes before the value of $\phi_{\text {ra }}$ goes through a minimum. In this case the value of $\phi_{r a}$ at the location where pellet multiplicity vanishes is the lowest value. Substituting eqs (9) and (10) into eq. (7) subject to $\theta_{a}=4 \theta_{f}\left(1+\theta_{f}\right)$ we find

$$
\phi_{r a, \min }=\frac{\psi-4(1+\Theta)}{\psi} \exp \left(\frac{\psi}{1+\Theta}-2\right)
$$

$\Theta$ being the dimensionless temperature at the location where pellet multiplicity vanishes.

(iii) No pellet multiplicity occurs in the reactor. Since eq. (11) is most stringent at the reactor entrance where $\Gamma=0$ and $\Theta=0$, this situation will occur if and only if

$$
\psi<4\left(1+\frac{L e^{2 / 3}}{\Delta \Theta_{a d}}\right) \Leftrightarrow \Delta \Theta_{a d}<\frac{4}{\psi-4} L e^{2 / 3}
$$

If we operate on the verge where pellet multiplicity disappears, the location where it vanishes is exactly at the reactor entrance. Hence, at this specific point $\phi_{\text {ra.min }}$ can be calculated from eq. (14) where, since we are positioned at $\omega=0$, we substitute $\Theta=0$, i.e.

$$
\phi_{\text {ra, } \min }=\frac{\psi-4}{\psi} \exp (\psi-2) \text {. }
$$

Now $\phi_{\text {ra, min }}$ is a function only of $\psi$ and does not depend anymore on $\Delta \Theta_{a d}, \Xi$ or Le.

\section{GENERALIZED CRITERIA FOR PELLET INSTABHLITY}

For given values of $\psi$ and $L e$ we can plot $\phi_{r a, \min }$ versus $\Delta \Theta_{a d}$ for several values of $\Xi$. A diagram is given in Fig. 2 for $\psi=10$, different values of $\Xi$ and for $L e=1$.

Independent of the value of $\Xi$, for every diagram similar to Fig. 2 there is a critical value of $\Delta \Theta_{a d}$ below which no pellet multiplicity can occur. As $\psi$ decreases (lower activation energies), this critical $\Delta \Theta_{a d}$ value increases according to eq. (15), i.e. the adiabatic temperature rise we can allow before pellets can become thermally unstable becomes higher as the activation energy is decreased. For $\psi=4$ this critical adiabatic temperature rise even passes the point of infinity so that for $\psi \leqslant 4$ we cannot ever have pellet instability, irrespective of the adiabatic temperature rise or the value of $\Xi$.

In the diagram of Fig. 2, if we have pellet multiplicity, $\phi_{r a, \min }$ decreases with increasing value of $\Delta \Theta_{a d}$ :

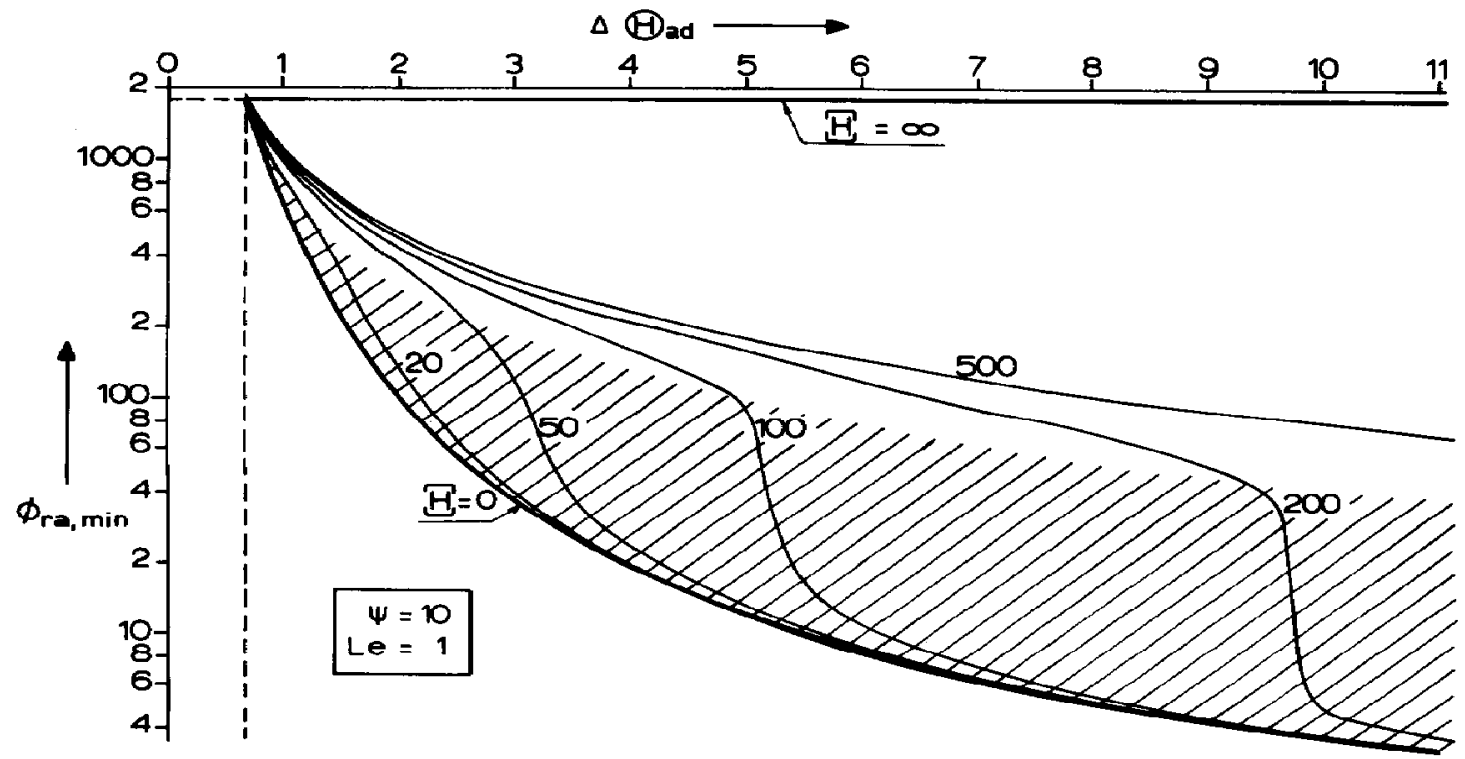

Fig. 2. The dimensionless minimum pellet size for which run away occurs in a packed bed; $\phi_{\mathrm{ra}, \mathrm{min}}$ versus the dimensionless adiabatic temperature rise $\Delta \Theta_{a d}$ for several ratios of the number of heat transfer units to the number of reaction units $\Xi=10$. Plots are given for an Arrhenius number $\psi=10$. Le $=1$ was assumed. 
as the adiabatic temperature rise increases we need smaller pellet sizes to create a large enough pellet specific heat transfer area and prevent run away of the pellet. For varying $\Xi$ values we have two ultimate regimes:

(i) For $\Xi \rightarrow \infty$ radial heat transfer is extremely fast and we have $\Theta=0$ everywhere in the reactor, i.e. the reactor is completely isothermal. For this situation it can be shown that $\phi_{\text {ramin }}$ is always found at the location where pellet multiplicity vanishes. Since also at this location $\Theta=0$, eq. (14) yields eq. (16) and $\phi_{\text {ra, min }}$ becomes independent of the values of $\Delta \Theta_{a d}$ and Le. Now $\phi_{\mathrm{ra}, \mathrm{min}}$ does not depend on $\Delta \Theta_{\text {ad }}$ anymore because the fluid temperature equals the coolant temperature anyway, irrespective of the value of $\Delta \Theta_{\text {ad }}$ -

(ii) If $\Xi=0$, there is no radial heat transfer whatsoever and we have an adiabatic packed-bed reactor. Values of $\phi_{\text {ra, min }}$ are lowest here because temperature profiles are most pronounced. Consequently $\phi_{\text {ra, min }}$ increases with increasing $\Xi$.

For given values of $\Delta \Theta_{a d}, \psi$ and $L e$ we cannot decrease the value of $\Xi$ at will. First of all, we also decrease the value of $\phi_{\mathbf{r a m} \text { min }}$ which favours pellet run away. Secondly, at some point we will enter the region of high parametric sensitivity, where reactor instability becomes a problem. These regions are indicated by the shaded areas in Fig. 2. The regions are a rough indication only, because we did not define parametric sensitivity properly. Notice that in the region of high parametric sensitivity the decrease of $\phi_{\text {ra, min }}$ with increasing $\Delta \Theta_{a d}$ is much more rapid than outside this region. This is because here small changes in the value of $\Delta \Theta_{a d}$ induce large changes in the value $\Gamma$ and $\Theta$ which in turn induce large changes in $\phi_{\text {ra, min }}$ -

\section{PELLET MULTIPLICITY AND REACTOR DESIGN}

For the design of a packed-bed reactor the choice of pellet size is usually based on the optimization of compression costs on the one hand, versus the costs of an increased reactor volume due to significant internal and/or external pellet mass transfer limitations, on the other hand. As a result of this procedure an optimum value $\phi_{\text {opt }}$ can be calculated according to

$$
\phi_{\text {opt }}=\frac{k_{0}}{k_{f}}\left(\frac{V_{p}}{A_{p}}\right)_{\text {denign }} .
$$

To investigate the significance of pellet thermal stability on reactor design the following recipe can be used:

(i) Calculate $\psi$ according to the Notation. If $\psi \leqslant 4$ pellet multiplicity cannot occur and need not be considered.

(ii) If $\psi>4$, calculate $\Delta \Theta_{a d}$ and estimate Le (usually Le $\approx 1$ ). When

$$
\Delta \Theta_{a d} \leqslant \frac{4}{\psi-4} L e^{2 / 3}
$$

pellet multiplicity cannot occur and need not be considered. (iii) If the last condition is not fulfilled, calculate $\phi_{\text {opt }}$ according to eq. (17). With the calculated values of $\psi$ and $\Delta \Theta_{a d}$ estimate the value of $\phi_{r a, \min }$ for $\Xi=0$, e.g. from Fig. 2 for $\psi=10$. If $\phi_{\text {opt }}$ is smaller than the value of $\phi_{r a, m i n}$, the pellet size is small enough to prevent pellet instability even for an adiabatic packedbed reactor and consequently pellet stability will not be significant.

(iv) If the last condition is not fulfilled, calculate $\Xi$. With this value the value of $\phi_{\text {ra, } m i n}$ must be estimated, e.g. from Fig. 2 for $\psi=10$. If $\phi_{\text {opt }}<\phi_{\text {ra, min }}$ the pellet size is sufficiently low to prevent pellet run away.

(v) If at the end of this procedure it turns out that $\phi_{\text {opt }} \geqslant \phi_{\text {ra, min }}$, then we cannot choose the optimum (i.e. most economical) size for the reactor pellets. Instead, a smaller size must be chosen to prevent thermal instability of the pellets. Of course, this will bring about higher compression costs, which in that case have to be accepted.

If Fig. 2 indicates that, for the calculated values of $\Xi$ and $\Delta \Theta_{a d}$ we operate in the shaded area, the reactor operates in the area of high parametric sensitivity and therefore the reactor becomes thermally instable. However, usually a tube diameter is selected in the following way. If the tube diameter becomes too large, due to thermal instability of the tube, a low conversion per pass must be accepted. This will bring about high recycling costs. On the other hand, if the tube diameter is chosen too small, the reactor investment will be very high. If this selection process is carried out properly, the reactor stability must have been considered and therefore the shaded area of Fig. 2 avoided.

\section{CONCLUSIONS}

When a pellet diameter is chosen based on the optimization of compression costs versus reactor investment, afterwards pellet thermal stability must be investigated. Four dimensionless numbers determine whether the pellets will be stable for the entire reactor. The generalized formulae and diagram discussed here allow a quick check of the significance of pellet stability.

Acknowledgements-These investigations are supported (in part) by the Netherlands' Foundations for Chemical Research SON with financial aid from the Netherlands' Technology Foundation STW.

\begin{tabular}{|c|c|}
\hline$a_{f}$ & thermal diffusivity of the fluid, $\mathrm{m}^{2} / \mathrm{s}$ \\
\hline $\begin{array}{l}\boldsymbol{A}_{p} \\
C_{f, \text { in }}\end{array}$ & $\begin{array}{l}\text { pellet external surface area, } \mathrm{m}^{2} \\
\text { concentration of the reactant in the fiuid } \\
\text { phase at the reactor entrance, } \mathrm{mol} / \mathrm{m}^{3}\end{array}$ \\
\hline$C_{f}$ & $\begin{array}{l}\text { concentration of the reactant in the fluid } \\
\text { phase, } \mathrm{mol} / \mathrm{m}^{3}\end{array}$ \\
\hline $\begin{array}{l}C_{p, S} \\
D\end{array}$ & $\begin{array}{l}\text { specific heat of the fluid, } J / \mathbf{k g ~ K} \\
\text { reactor diameter, } \mathrm{m}\end{array}$ \\
\hline $\mathbb{D}_{f}$ & $\begin{array}{l}\text { diffusion coefficient of the reactant in the } \\
\text { fluid phase, } \mathrm{m}^{2} / \mathrm{s}\end{array}$ \\
\hline$E_{a}$ & activation energy, $\mathrm{J} / \mathrm{mol}$ \\
\hline
\end{tabular}

\section{NOTATION}


$(-\Delta H)$

$\boldsymbol{k}_{\mathrm{c}}$

$\boldsymbol{k}_{\mathbf{0}}$

$\boldsymbol{k}_{\boldsymbol{f}}$

$L$

Le

NRU

NTU

$\boldsymbol{R}$
$T_{c}$
$T_{f}$
$T_{p}$
$U$

$v_{0}$

$V_{p}$

$\boldsymbol{z}$

Greek letters

$\alpha_{f}$

$\Gamma$

$\boldsymbol{\varepsilon}$

$\theta_{a}$

$\theta_{f}$

$\theta_{p}$ reaction enthalpy, $\mathrm{J} / \mathrm{mol}$ reactant

reaction rate constant based on the catalyst volume at coolant temperature, $s^{-1}$

pre-exponential factor based on the catalyst volume, $\mathrm{s}^{-1}$

mass transfer coefficient for a pellet, $\mathrm{m} / \mathrm{s}$ reactor tube length, $m$

Lewis number of the reactant $\left(=a_{f} / \mathbb{D}_{f}\right)$, dimensionless

number of reaction units $\left[=k_{c} L(1\right.$ $\left.-\varepsilon) / v_{0}\right]$, dimensionless

number of heat transfer units $(=4 U L /$ $\left.\rho_{f} C_{p, f} v_{0} D\right)$, dimensionless

ideal gas constant, $8.31434 \mathrm{~J} / \mathrm{mol} \mathrm{K}$

coolant temperature, $\mathbf{K}$

fluid temperature, $\mathbf{K}$

temperature of the catalyst pellet, $K$

overall radial heat transfer coefficient, $\mathbf{W} / \mathbf{m}^{2} \mathbf{K}$

superficial fluid velocity based upon the empty reactor tube, $\mathrm{m} / \mathrm{s}$

pellet volume, $\mathrm{m}^{3}$

axial coordinate, $m$

heat transfer coefficient between the pellet and the fluid, $W / \mathrm{m}^{2} \mathrm{~K}$

dimensionless reactant concentration, conversion $\left[=\left(C_{f, \text { in }}-C_{f}\right) / C_{f, \text { in }}\right]$, $\mathrm{di}$ mensionless

porosity of the packed bed, volume fraction of the fluid, dimensionless

dimensionless activation temperature $\left\{=\left[\rho_{f} C_{p, f} E_{a} /(-\Delta H) C_{f} R\right] L e^{2 / 3}\right\}$, dimensionless

dimensionless fluid temperature $\left\{=\left[\rho_{f} C_{p, S} T_{f} /(-\Delta H) C_{f}\right] L e^{2 / 3}\right\}, \quad$ dimensionless

dimensionless pellet temperature $\left\{=\left[\rho_{f} C_{p . f} T_{p} /(-\Delta H) C_{f}\right] L e^{2 / 3}\right\}, \quad$ dimensionless
$\Theta$

$\Delta \Theta_{a d}$

$\Xi$

$\rho_{f}$

$\phi$

$\psi$

$\omega$

\section{REFERENCES}

Eigenberger, G., 1972, Dynamic behaviour of the catalytic fixed-bed reactor in the region of multiple steady states. I. Influence of heat conduction in two phase models. Chem. Engng Sci. 27, 1909-1915.

Liu, S. L. and Amundson, N. R., 1962, Stability of adiabatic packed bed reactors - elementary treatment. Ind. Engng Chem. Fundam. 1, 200-208.

Liu, S. L. and Amundson, N. R., 1963, Stability of adiabatic packed bed reactors-effect of axial mixing. Ind. Engng Chem. Fundam. 2, 183-189.

Liu, S. L., Aris, R. and Amundson, N. R., 1963, Stability of non-adiabatic packed bed reactors. An elementary treatment. Ind. Engng Chem. Fundam. 2, 12-20.

Morbidelli, M. and Varma, A., 1986, Parametric sensitivity in fixed-bed catalytic reactors: the role of inter-particle resistances. A.I.Ch.E. J. 32, 297-306.

Morbidelli, M. and Varma, A., 1987, Parametric sensitivity in fixed-bed catalytic reactors: inter- and intraparticle resistance. A.I.Ch.E. J. 33, 1949-1958.

Westerterp, $\mathbf{K}$. R., van Swaaij, W. P. M. and Beenackers, A. A. C. M., 1987, Chemical Reactor Design and Operation. Wiley, Chichester. 